\title{
A Clinical Study and Management of Varicocele
}

\author{
Dr S Abijith Sudhakar Shetty ${ }^{1}$, Dr Balaji Prabakaran ${ }^{2}$, Dr Bharath ${ }^{3}$
}

\begin{abstract}
Background Data: Varicocele, an abnormal dilatation and tortuosity of the pampiniformplexes of veins is found in about $15 \%$ of the general population with a marked left ed predominance. However this incidence rises to $35 \%$ in men with primary fertility and 70 to $80 \%$ in men with secondary infertility. Although varicocelectomy is the most commonly performed procedure for correction of male subfertility with seminal imp nent in 60 to $80 \%$ of the subjects and pregnancy rates ranging from 20 to $60 \%$ there is still no consensus on the best method of performing the varicocelectomy. Materials and Methods: A prospective study of 50 adult patients suffering from unilateral/bilateral variccele presenting with infertility or pain. All the patients included in the study unit went varicocelcectomy in the period ranging between May 2006 and Sept 2008 and their ages ranged from 18 yrs to 50 . Results: The operating time was found to be nearly same for open $(46.04 \mathrm{~min})$ and laparoscopic $(45.4 \mathrm{~min})$ varicocelectomy in bilateral cases. Laparoscopic approach also provided the advantage of being performed through the same ports for both sides. However for unilateral varicocelectomy, operating time was much shorter for open procedures (23.02min to 40.66min). The postoperative pain score calculated over the first 3 postoperative days was found to be higher in the open group (6) compared to laparoscopic group (3.94). Among the open group, postoperative pain was found to be greatest with the inguinal group (6.54) compared to palomo group (5.85). Symptomatic relief in patients operated for painful varicocele was found to be better achieved by open (64.29\%) than the laparoscopic approach (57.15\%). Among the inguinal approach was found to give better results with relief of pain on $67.66 \%$ of the patients whereas the palomo's approach yielded success in $50 \%$ of patients. Improvement in seminal parameters was found to be achieved better with open (69.66\%) than laparoscopic (62.5\%) approaches and inguinal (71.43\%) better than palomo's approach (57.15\%). Conclusion: Varicocelectomy has been performed as a procedure for correction of male sub fertility for a long time. Several uncontrolled studies have shown improvement in the seminal parameters after varicocelectomy and also enhanced pregnancy rates. The findings of our study were consistent with the above mentioned established facts. It was found that varicocelectomy performed by any method led to an improvement in seminal parameters and pregnancy rates in properly selected cases. There was significant difference in the operating time between open and laparascopic approaches for unilateral varicocelectomy. However, for bilateral varicocelcectomy operating time for the open or laparascopic procedure remains nearly the same. The laparascopic approach also provides the advantages of being able to be performed through the same ports for both sides thus avoiding additional incisions. Improvement in seminal parameters was noted in $62.5 \%$ of patients after laparoscopic varicocelectomy and pregnancy achieved in $32.5 \%$ of the patients which were comparable to the results obtained with open varicocelectomy. Thus, varicocelectomy in experienced hands could be considered as a good option for patients being considered for bilateral varicocelectomy over open varicocelectomy. Among the open approaches, the inguinal approach (66.66\%) fared better than the Paloma's approach (50\%).
\end{abstract}

Keywords: varicocelectomy, palomo's, Varicocele,infertility, hydroceles.

\section{Introduction}

Varicocele, an abnormal dilatation and tortuosity of the pampiniform plexus of veins is found in about $15 \%$ of the general population with a marked left sided predominance. However this incidence rises to $35 \%$ in men with primary infertility and 70 to $80 \%$ in men with secondary infertility. Mounting evidence clearly indicates that varicoceles cause progressive size and duration dependent testicular damage. Although varicocelectomy is the most commonly performed procedure for correction of male subfertility with seminal improvement in 60 to $80 \%$ of the subjects and pregnancy rates ranging from 20 to $60 \%$ there is still no consensus on the best method of performing the varicocelectomy.

There are many surgical approaches to repairing varicoceles including retroperitoneal, inguinal, sub inguinal, scrotal and most recently laparoscopic techniques with each technique having their own set of advantages and disadvantages.

Each of the open approaches have their share of proponents, the inguinal and retroperitoneal techniques being more popular. The advent of laparoscopic varicocelectomy in the late 1980's and early 90's added another contender to the already pre existing argument over the best technique for variocelectomy. The laparoscopic approach is essentially an artery sparing retroperitoneal procedure with the same disadvantages as the open retroperitoneal approach. The laparoscopic approach however converted the retroperitoneal approach into an intra peritoneal approach and also introduced its own unique set of complications including bowel injury, air embolism etc and also had to be performed under G.A. A number of reports however have demonstrated the efficiency and low morbidity of the procedure.

We decided to conduct a study both prospective and retrospective comparing the various open and laparoscopic approaches to varicocelectomy to try and predict the best possible approach with regard to outcome, complications and recurrence rates in our set up.

\section{Materials and Methods}

Methods of study: This is a prospective study of patients suffering from unilateral/bilateral varicocele presenting with infertility or pain was carried out at Yenepoya Medical college Hospital Mangalore.

Among the 50 patients, 15 underwent laparoscopic varicocelectomy whereas the other 35 underwent open varicocelectomy, out of which 15 by inguinal approach, 15 by reteoperitoeneal approach and 5 subguinal approach. All the patients included in the study underwent varicocelcectomy in the period ranging between May 2006 and Sept 2008 and their ages ranged from 18 yrs to 50 yrs. 


\section{International Journal of Science and Research (IJSR) ISSN (Online): 2319-7064 \\ Index Copernicus Value (2013): 6.14 | Impact Factor (2015): 6.391}

\section{Methodology}

A detailed history taking and clinical examination of the patient was carried out at the time of admission. The clinical suspicion of varicocele was confirmed with the use of scrotal ultrasound Doppler study. The varicocele were graded into Grades I, II or III on the basis of the USG and the diameter of the veins was also noted. A semen analysis of the patient was obtained in patients presenting with infertility. the choice of approach (i.e open or laparascopic) was left to the patient. The patients preferring open varicocelectomy were randomly allocated to the inguinal or palomo group. All the open procedures were performed under spinal anesthesia and all laparoscopic procedures under general anaethesia. The laparoscopic procedure was performed using 3 or 4 ports. A $10 \mathrm{~mm}$ umbilical port and another $5 \mathrm{~mm}$ port midway between the umbilicus and the public symphisis in the midline was common to all procedures. Further choice was between a $5 \mathrm{~mm}$ or $10 \mathrm{~mm}$ port laterally. If the spermatic veins were tackled by clip ligation then it necessitated the use of a $10 \mathrm{~mm}$ port laterally. The skin incisions were about $5 \mathrm{~cm}$ for inguinal, $3 \mathrm{~cm}$ for palomo's and 2cm for sub inguinal varicocelectomy. The veins were isolated in each of the procedures, ligated with vicryl and a segment of the veins excised between ligatures.

\section{Sample Size:}

\section{CASES}

\section{Results}

Table 1: Number of cases and the approaches

\begin{tabular}{|l|c|c|c|}
\hline & & Open & Laparoscopic \\
\hline No. of cases & Inguinal & 15 & \multirow{2}{*}{} \\
\hline & Palomo's & 15 & \multirow{2}{*}{15} \\
\hline & Subinguinal & 5 & \multirow{2}{*}{15} \\
\hline & Total & 35 & 7 \\
\hline Indication & Pain & 16 & 7 \\
\hline & Infertility & 19 & 8 \\
\hline
\end{tabular}

The operating time was found to be nearly same for open (46.04min) and laparoscopic (45.4min) varicocelectomy in bilateral cases. Laparoscopic approach also provided the advantage of being performed through the same ports for both sides. However for unilateral varicocelectomy, operating time was much shorter for open procedures (23.02min to $40.66 \mathrm{~min}$ ). this is because most of the time consumed during the laparoscopic procedure was for instrumentation and gas insufflations, the actual operating time being rather short. Among the open group, time consumed by any of the approaches was almost similar.

The postoperative pain score calculated over the first 3 postoperative days was found to be higher in the open group (6) compared to laparoscopic group (3.94). Among the open group, postoperative pain was found to be greatest with the inguinal group (6.54) compared to palomo group (5.85). The patients operated by subguinal approach had a pain score of (4.75) but the reliability of this figure may be doubtful due to the small number of patients in this group (4). consumed by the patients in the first post operative week, number of days of hospital stay and number of days for return to normal activity.

There was no instance of serious intraoperative or postoperative complications under the open or Laparoscopic group. Symptomatic relief in patients operated for painful varicocele was found to be better achieved by open (64.29\%) than the laparoscopic approach (57.15\%). Among the inguinal approach was found to give better results with relief of pain on $67.66 \%$ of the patients whereas the palomo's approach yielded success in $50 \%$ of patients.

Improvement in seminal parameters was found to be achieved better with open (69.66\%) than laparoscopic (62.5\%) approaches and inguinal (71.43\%) better than palomo's approach (57.15\%). The pregnancy rates achieved also showed a similar pattern as shown in the comparative tables.

Cost was a major inhibitory factor in the laparoscopic group with the patient having to bear about 1.5 to 3 times more than the expenditure for open varicocelectomy by any approach

Occurrence of post operative hydrocele was a feature noted only with the inguinal (15.38\%) and subguinal (50\%) approaches. However all the hydroceles noticed under our study resolved spontaneously over a period of time.

The inguinal and subguinal approaches however fared better than the other approaches as there were no recurrences at least in the duration of our study. The recurrence rates in the palomo's group was $15.38 \%$ where as it was $13.33 \%$ in the laparoscopic group.

Table 2: Open Vs Laparoscopic Varicocelectomy

\begin{tabular}{|c|c|c|c|c|}
\hline & & & \begin{tabular}{|c|} 
Open \\
varicocelectomy \\
\end{tabular} & \begin{tabular}{|c|} 
Laparoscopic \\
varicocelectomy
\end{tabular} \\
\hline \multirow[t]{2}{*}{1.} & Duration of surgery & $\mathrm{B} / \mathrm{L}$ & 46.04 & 45.4 \\
\hline & & $\mathrm{U} / \mathrm{L}$ & 23.02 & 40.66 \\
\hline 2. & \multicolumn{2}{|c|}{$\begin{array}{l}\text { Post operative pain score } \\
\text { (over first } 3 \text { post operative } \\
\text { days) }\end{array}$} & $\begin{array}{c}6 \\
(0.83)\end{array}$ & $\begin{array}{c}3.94 \\
(0.258)\end{array}$ \\
\hline \multirow[t]{2}{*}{3.} & \multicolumn{2}{|l|}{$\begin{array}{l}\text { Number of analgesic } \\
\text { tablets }\end{array}$} & 12.97 & 8.8 \\
\hline & \multicolumn{2}{|c|}{$\begin{array}{l}\text { Consumed in the first post } \\
\text { Operative week }\end{array}$} & (1.938) & $(0.414)$ \\
\hline 4. & \multicolumn{2}{|c|}{$\begin{array}{c}\text { Number of days of } \\
\text { hospital stay }\end{array}$} & $\begin{array}{c}2.9 \\
(0.548)\end{array}$ & $\begin{array}{c}2 \\
(0)\end{array}$ \\
\hline 5. & \multicolumn{2}{|l|}{ Cost } & + & ++ \\
\hline 6. & \multicolumn{2}{|c|}{$\begin{array}{l}\text { Number of days for return } \\
\text { to normal activity }\end{array}$} & $\begin{array}{c}6.9 \\
(1-155) \\
\end{array}$ & $\begin{array}{c}4 \\
(0) \\
\end{array}$ \\
\hline 7. & \multicolumn{2}{|c|}{ Serious complications } & - & - \\
\hline 8. & \multicolumn{2}{|c|}{ Symptom relief } & $64.29 \%$ & $57.15 \%$ \\
\hline 9. & \multicolumn{2}{|c|}{$\begin{array}{c}\text { Improvement in seminal } \\
\text { parameters }\end{array}$} & $69.66 \%$ & $62.5 \%$ \\
\hline 10. & \multicolumn{2}{|c|}{ Pregnancy achieved } & $37.5 \%$ & $37.5 \%$ \\
\hline 11. & \multicolumn{2}{|c|}{ Post operative hydrocele } & $13.33 \%$ & 0 \\
\hline 12. & \multicolumn{2}{|c|}{ Recurrence rate } & $6.66 \%$ & $13.33 \%$ \\
\hline
\end{tabular}

A trend similar to the one seen with regards to the pain score was seen in terms of the number of analgesic tablets 


\section{International Journal of Science and Research (IJSR) \\ ISSN (Online): 2319-7064}

Index Copernicus Value (2013): 6.14 | Impact Factor (2015): 6.391

Table 3: InguinalsVsPalomo'sVaricocelectomy

\begin{tabular}{|l|l|c|c|}
\hline & & Inguinal & Palomo's \\
\hline 1. & Duration of surgery (min) (U/L) & 22.25 & 21.65 \\
\hline 2. & Post operative pain score & $6.54(0.66)$ & $5.85(0.37)$ \\
\hline 3. & No. of analgestic tablets & 13.85 & 13.08 \\
& Consumed & $(1.77)$ & $(0.76)$ \\
\hline 4. & No. of days of hospital stay & $2.8 \wedge 5(0.38)$ & $2.85(0.38)$ \\
\hline 5. & No. of days of return to & 7.62 & 6.46 \\
& normal activity & $(1.26)$ & $(0.52)$ \\
\hline 6. & Serious complication & 0 & 0 \\
\hline 7. & Cost & - & $=$ \\
\hline 8. & Symptom relief & $67.66 \%$ & $50 \%$ \\
\hline 9. & Improvements in seminal parameters & $71.43 \%$ & $57.15 \%$ \\
\hline 10. & Pregnancy achieved & $42.86 \%$ & $29.57 \%$ \\
\hline 11. & Postoperaive hydrocele & $15.38 \%$ & 0 \\
\hline 12. & Recurrence & 0 & $15.38 \%$ \\
\hline
\end{tabular}

\section{Discussion}

Varicocelectomy has been performed as a procedure for correction of male sub fertility for a long time. Several uncontrolled studies have shown improvement in the seminal parameters after varicocelectomy and also enhanced pregnancy rates. The findings of our study were consistent with the above mentioned established facts. It was found that varicocelectomy performed by any method led to an improvement in seminal parameters and pregnancy rates in properly selected cases.

There was significant difference in the operating time between open and laparascopic approaches for unilateral varicocelectomy. However, for bilateral varicocelcectomy, as is universally practiced in patients being operated for infertility the operating time for the open or laparascopic procedure remains nearly the same. This is probably because most of the time consumed during the laparoscopic approach is for instrumentation which is common for both sides. The laparascopic approach also provides the advantages of being able to be performed through the same ports for both sides thus avoiding additional incisions. Improvement in seminal parameters was noted in $62.5 \%$ of patients after laparoscopic varicocelectomy and pregnancy achieved in $32.5 \%$ of the patients which were comparable to the results obtained with open varicocelectomy.

Thus, laparoscopic varicocelectomy in experienced hands could be considered as a good option for patients being considered for bilateral varicocelectomy over open varicocelectomy are reflected in a number of parameters which were assessed during the study such as the postoperative pain score, the number of analgesic tablets consumed by the patient in the first post operative week, the number of days of hospital stay and the number of days for return to normal activity. The laparoscopic approach was found to be superior to the open approach in all the above regards and the statistical significance of these findings were also established. With open varicocelectomy, improvement in seminal variables was noticed in $69.66 \%$ of patients with achievement of pregnancy in $37.5 \%$ of patients.

The principal draw back of the laparoscopic approach is the higher recurrence rate and a potential for serious complications. The laparoscopic approach is essentially a high ligation of the internal spermatic vein performed under magnification which provides the advantage of safe guarding the testicular artery and lymphatic channels more easily. But, since the external spermatic veins mainly the cremasteric vein is not taken it can lead to higher rates of recurrence. This is however not very significant in cases operated for infertility as there is always improvement in the venous hypertension and reflux following surgery as reflected in the improved seminal parameters and pregnancy rates post operatively. This is because the recurrent varicocele which develops due to communication with the patent external spermatic venous system takes some time to develop. This however assumes much greater significance in the instance of a symptomatic varicocele as will be subsequently discussed.

Another drawback of the laparoscopic approach is a potential for serious complications as it converts an extra peritoneal procedure into an intraperitoneal one. However the actual incidence of these complications is to a great extent operator dependent and in experienced hands seems to be negligible. Another factor is that laparoscopic varicocelectomy has to be performed under general anaesthesia whereas spinal anaesthesia is preferred for all forms of open varicocelectomy thus precluding medical fitness of the patient for general anaesthesis as a factor for undergoing laparoscopic varicocelectomy. In our study there was one incidence of severe bronchospasm but the patient recovered to normalcy with medical management.

Among the various methods jof open varicocelectomy the immediate post operative morbidity in terms of the postoperative pain and time to recovery was found to be greatest with the inguinal approach and least with the subinguinal approach and with the Palomo's approach intermediate. The subinguinal approach was however used in only 4 cases and hence is not being considered for comparison. The increased postoperative pain and morbidity with the inguinal approach was probably because of opening up of external oblique aponeurosis and the cremasteric box. This is best demonstrated by the fact that the pain is much lesser in the sub inguinal approach where the veins are tackled at the superficial inguinal ring without opening up the external oblique aponeurosis. However, the inguinal approach scores over the Palomo's approach in that the cremasteric vein is always looked for and then ligated and this is probably the reason for the absence of any recurrence in the inguinal group as compared to $15.38 \%$ recurrence in the Palomo's group. The external spermatic veins are tackled in the subinguinal approach as well and even here no recurrences were found although the numbers of cases were limited.

The incidence of post operative hydrocele was found to be higher in the inguinal group where no hydroceles were found in our study. This could possibly be explained by the fact that the cremasteric box is opened up in the inguinal approach. The number of veins is more, their diameters being smaller and damage being caused to the lymphatic channels when attempts are made to isolate the veins before ligation them. Another significant factor is that with ligation of the cremasteric vein, the lymphatic channels running along both sets of venous system may be damaged thus 


\section{International Journal of Science and Research (IJSR) \\ ISSN (Online): 2319-7064 \\ Index Copernicus Value (2013): 6.14 | Impact Factor (2015): 6.391}

causing a higher incidence of postoperative hydrocele. It has been proved historically that the post operative hydrocele is due to lymphatic obstruction. In most of the cases, however as seen in all the cases of postoperative hydrocele in our study there is spontaneous resolution over a period of time as lymphatic collaterals develop. There were no incidences of post operative hydroceles in the laparoscopic and Palomo's group in your study probably because of the larger size and the lesser number of veins found and also because of the additional advantage of magnification provided by the laparoscopic approach. This is further substantiated by the findings in several studies world wide that application of microsurgical techniques to the inguinal and subinguinal approaches tends to reduce the incidence of post operative hydrocele.

Symptomatic relief in patients operated for painful varicocele was found to be better achieved by open (64.29\%) than the laparoscopic approach (57.15\%).

Among the open approaches, the inguinal approach (67.66\%) fared better than the Palomo's approach (50\%). This can only the explained by the additional manouvre of ligation of the external spermatic system of veins in the form of the cremasteric vein in the inguinal approach. When this findings is coupled with the incidence of higher recurrence rates in the Palomo and laparoscopic groups as compared to the inguinal group as mentioned earlier it would suggest that the inguinal approach seems to be aj better approach when compared to the other two at least in the setting of symptomatic varicocele. It was also found that anchoring of the distal bunch of veins after ligation to the external oblique aponeurosi, provides some kind of additional mechanical support which seemed to produce better symptomatic relief but the exact significance of this finding needs to be further investigated.

The findings in the subinguinal group were very impressive with $100 \%$ symptomatic relief, no recurrence and 50\% pregnancy rates But, as the study group was very small (4 cases) drawing any conclusions from these findings were not prudent.

In the comparison between the open and laparoscopic approaches cost is an important inhibitory factor with the laparoscopic approach costing 1.5 to 2 times more.

Proper case selection was found to be an important factor in determining the post operative results achieved following varicocelectomy both in infertility based and symptomatic varicoceles. It was found in our study that greater the size/grade of the varicocele, greater the degree of improvement after varicocelectomy. This was reflected both in the improvement in general parameters and pregnancy rates as well as the symptomatic relief following varicocelectomy.

Results were poor in the absence of a clinically demonstrable varicocele or grade I varicoceles on Doppler examination with veins $<2 \mathrm{~mm}$ in diameter and the absence of demonstrable reflux. It was seen that in most of these cases the infertility or the pain experienced was usually a result of some other pathology and showed no improvement by a varicocelectomy Especially in a case of infertility in inconclusive or doubtful cases with regards to varicocele as the cause, it seems prudent to confirm the normal functioning of the Hypothalamus - Pituitary - Gonadal axis by investigating for serum levels of Gonadotropins ( $\mathrm{LH}$ and FSH) and may be even testosterone before subjecting the patient to an unnecessary varicocelectomy. Another finding in the study was that the patients whose pre operative seminal parameters were in the mid range with regards to the sperm count and motility were found to show a better response to varicocelectomy than the patients having very low counts and motility in the preoperative semen analysis.

\section{Summary and Conclusion}

1) In our study, 50 patients with varicocele are of younger age group and their main presenting complaint is pain.

2) Laparoscopic varicocelectomy is more time consuming than open procedures. However this differences is limited to unilateral surgeries. In the insistence of bilateral varicocelectomy the time taken is nearly the same for open and laparascopic procedures.

3) The intraperitoneal approach and general anaesthesia required for laparoscopy pose the risk of far severe complications than open procedures although the incidence of these major complications is negligible.

4) Post operative pain is significantly higher in any of the open procedures compared to the laparoscopic approach. Among the open procedures the inguinal approach was found to be the most notorious where as the subinguinal approach tended to cause the least postoperative pain. A similar pattern was also reflected in the return to normal activity post operatively.

5) In cases operated for infertility, varicocelectomy performed through any approach tended to produce an improvement in the seminal parameters in the form of increased count and motility and decreased number of abnormal forms. Individual having seminal parameters in the mid range showed a better improvement

6) The recurrence rates were found to be higher with laparoscopic and palmo's approach than with inguinal or subinguinal varicocelectomy. This difference was probably due to the tackling of the cremasteric veins in the latter approaches.

7) Postoperative hydrocele was seen as an occasional complication of inguinal and subinguinal varicocelectomy. All the hydroceles resolved spontaneously in our study.

\section{Referrences}

[1] Gorelick J, Goldstein. Loss of fertility in men with varicocele,Fertility and sterility.1993; 59(3):613-6.

[2] LipshultzL.Varicocele a progressive or static lesion?Urology. 1993; 42(5): 541 - 3.

[3] Schelsinger M H, Wilets I F,Nagler H M.Treatment outcome after varicocelectomy , a critical analysis.Urology Clinics of North America. 1994;21 (3):517-29.3

[4] Spencer W G. Varicocele anatomy, Journal of urology. 1992; 148(4): 1190-4 
[5] Curling TB. A practical treatise on the diseases of testis and of the spermatic cord and scortum. Philadelphia. 1856;23(4):67-70

[6] Barwell R, One hundred cases of varicocele treated by the subcutaneous wire loop. Lancet Journal of urology. 1885; 1:978.

[7] Bennet W H. Varicocele particularly with reference to its radical cure Lancet Journal of Urology.1889;1:261.

[8] Macomber D, Sanders M B. The spermatozoa count, its value in the diagnosis, prognosis and treatment of sterility. New England Journal of Medicine. 1929;200:981

[9] Tulloch W S. Varicocele in subfertility Results of treatment. British Journal of Medicine.1944;2:356.

[10] Madgar I, Weissenberg R, Lunefield B et al . Testicular veins anatomy and role in varicocelogenesis and other pathologic conditions, Urology. 1990;35(2):175 - 82.

[11] Shafik A, Mofathah A, Olfat S et al .Testicular veins anatomy and role in varicocelogenesis and other pathologic conditions .Urology 1990;35 (2):175-82.

[12] Turner T, Howard S. venous anatomy of the left sided varicocele Fertility and sterility. New England Journal of Medicine. 1994; 62(4): 869-875.

[13] Saypol D C, Lipshuitz L I, Howard S S .Varicocele. Journal of Andrology 1981;2:61.

[14] Coolset B L R A .The varicocele syndrome venography for determining the optional level for surgical management. Journal of Urology 1980;124 833.

[15] Wishahi M M . Detailed anatomy of the internal spermatic vein and ovarin vein, Human cadaver study and operative spermatic venography : clinical aspects, Journal of Urology. $1991 ; 145(4): 780-4$.

[16] Beck E M, Schelgel P N, Goldstein M .Intraoperative varicocele anatomy a macroscopic and microscopic study.Journal of Urology. 1992; 148(4):1190-4.

[17] Sigman M, Howard S S. Male infertility Cambells Urology. 1997;2: 1287- 330.

[18] Paduch D A, Skoog S J.Current Management of adolescent varicocele. Rev Urology.2001;3(3):120-33

[19] AgarwalS.Vascular morphology in testes of infertile males with varicocele. Indian Journal of Medicine.1991;94:228-31.

[20] Tanji N, Fujiwara T, Kaji H et al.Histologic evaluation of spermatic veins in patients with varicocele,Indian Journal of Urology. 1999;6(7): 355-60. 